\title{
Discovering the Keys: Transformative and Translational Mechanobiology
}

The 2018 Cellular and Molecular Bioengineering (CMBE) conference, which is the annual meeting for the CMBE special interest group (SIG) of the Biomedical Engineering Society (BMES), will be held at the beautiful Ocean Reef Club in Key Largo, FL, from January 2-6, 2018. The theme of this year's conference is Discovering the Keys: Transformative and Translational Mechanobiology. We put together an outstanding program of keynote and invited speakers from engineering, biology, and medicine who will present the latest research covering both breadth and depth in mechanobiology. In addition, we will recognize outstanding abstract submissions with 'Rising Star' Awards for early stage investigators and 'Shooting Star' Awards for graduate students and post-doctoral fellows. Finally, we will present the Shu Chien Achievement award, bestowed upon an individual who has demonstrated meritorious contributions to the field of cellular and molecular bioengineering, including groundbreaking scientific advances, program development, and mentoring and training of the next generation of scientists in the field of cell and molecular bioengineering. Previous awardees include Douglas Lauffenburger (2015), Don Ingber (2016), and Antonios Mikos (2017).

The conference theme was selected to address the key challenge in how mechanobiology can continue to advance the study of pathophysiology and improve human health. It is now well accepted that both the mechanical properties of biological systems and mechanical forces applied to biological systems play critical roles in pathology and pathophysiology. Important challenges remain in creating new technologies to probe and manipulate biomechanics in vivo and use these discoveries to enhance human health. To date, isolated groups of investigators from diverse fields have explored mechanobiology research but without widespread communication across individual groups, much less a common vision for how to encourage clinical translation. There is a need for a unique and dedicated platform to bring these complementary groups of investigators together. We planned a conference integrating engineers and biologists with clinical and/or entrepreneurial experience, each of whom has started to bridge the gap between fundamental research and clinical translation. The outcome of the conference will be increased technology sharing amongst mechanobiology researchers, as well as a new vision and strategic plan for overcoming clinical translation gaps in mechanobiology.

The conference sub-themes will highlight emerging mechanobiology research areas, including: mechanobiology of morphogenesis and development; multiscale mechanobiology of disease; systems mechanobiology and heterogeneity; mechanophenotyping and high-throughput diagnostics; modulating stem cell mechanobiology in regenerative medicine; and novel tools in mechanobiology - sensors, biomaterials, and gene editing. Invited speakers and selected speakers from the submitted abstracts will show how their research bridges the gap between bench and bedside in mechanobiology. For example, our first keynote speaker, Dr. Cato Laurencin, is both an engineer and a surgeon. He is the Chief Executive Officer of the Connecticut Institute for Clinical and Translational Science and the Director of the Institute for Regenerative Engineering at the University of Connecticut. During his keynote presentation, Dr. Laurencin will share his insight into the field of regenerative engineering, which is the convergence of advanced materials science, stem cell science, physics, developmental biology, and clinical translation. Another highlighted keynote speaker is Dr. Molly Stevens, Professor of Biomedical Materials and Regenerative Medicine at Imperial College in London. Dr. Stevens' research spans fundamental material science and translational studies to improve human health, including the exploration of dynamic nanomaterials as biosensors and as advanced tissue engineering scaffolds that direct cell-material interactions. Additional keynote speakers include Dr. Dennis Discher, Dr. Farshid Guilak, Dr. Melody Swartz, and Dr. Todd McDevitt.

We also planned many sessions to encourage interactions among engineers, biologists, and clinicians at all levels. The CMBE conference follows the Gordon conference model, with a single track of podium sessions populated primarily by invited talks from leaders in mechanobiology research. Early stage investigators will be highlighted during the 'Rising Star' podium session, which will take place on the first day of the conference to enhance exposure and facilitate networking with senior investigators. Top abstracts from post-doctoral fellows and graduate students will be incorporated into podium presentations and highlighted in poster sessions, which will take place during 
morning session coffee breaks. These recognized speakers will receive travel awards to defray the cost of attending and presenting at the conference. Program managers from the National Science Foundation and National Institutes of Health will lead a grant writing workshop. In addition to informal interactions via conference-organized social activities, invited speakers and leaders will provide mentoring opportunities to early stage investigators, post-doctoral fellows and graduate students through a "Lunch with Leaders" program. Finally, the conference will close with a session dedicated to creating strategies for translating mechanobiology to improve human health, with a panel discussion of our keynote speakers moderated by an expert in translational medicine.

The CMBE conference builds on the $30+$ year history of the successful Society for Physical Regulation in Biology and Medicine (SPRBM) conferences. Each CMBE conference has a different theme, making the annual meeting a unique event for the dissemination of discoveries in a CMBE subtopic. As conference organizers, we study mechanobiology in different physiological systems (orthopedics, development, cardiovascular) and at different length scales (molecular, cellular, tissue). We put together this conference to employ 'creative destruction' of the barriers that separate mechanobiology research in disparate physiological systems and length scales. We believe that mechanobiological understanding can benefit from discoveries and techniques used in different physiological systems or at different length scales. Integrated thinking in this area can then be harnessed and applied to further drive the integration of mechanobiology into the fields of cell biology, drug discovery, and regenerative medicine.

We would like to thank (in advance) the CMBE SIG council members for assistance in program planning, reviewing abstracts, and selecting awardees and speakers; BMES President Lori Setton, CMBE SIG Chair Roland Kaunas, and the BMES Board of
Directors and CMBE SIG Council for helping to organize and execute this conference; and the BMES Staff, especially Terry Young, for their time and effort to make this event a success. We are also delighted to have the support of numerous agencies and corporate sponsors.

We hope you find the $2018 \mathrm{CMBE}$ conference intellectually stimulating, with opportunities to highlight your current research, forge new collaborations, and develop pathways to translate mechanobiology research into the clinic. We invite you to attend the meeting and submit your mechanobiology research for presentation. The abstract submission deadline is August 28, 2017. Additional information about this conference is available online: http://www.bmes.org/ cmbeconference18. We hope that you will join us in Florida this winter!

\section{Alisa Morss Clyne}

Mechanical Engineering and Mechanics, School of Biomedical Engineering,

Science and Health Systems,

Drexel University, Philadelphia, USA

Electronic mail: asm67@drexel.edu

ERIC M. DARLING

Department of Molecular Pharmacology, Physiology, and Biotechnology, Department of Orthopaedics, Center for Biomedical Engineering, School of Engineering, Brown University, Providence, USA

Nadeen O. Chahine

Department of Orthopedic Surgery, Department of Biomedical Engineering, Columbia University, New York, USA 